\title{
Ontology: A Support Structure for a V-Labs Network: Euronet-Lab
}

\author{
http://dx.doi.org/10.3991/ijoe.v8iS3.2257 \\ C.C. Raúl ${ }^{1}$, M.F. José 2 and D. Andrew ${ }^{3}$ \\ ${ }^{1}$ EST - Instituto Politécnico de Setúbal, Setúbal, Portugal \\ ${ }^{2}$ FCT - Univ. Nova de Lisboa, Caparica, Portugal \\ ${ }^{3}$ ITT - Inst. Technology Tallaght, Dublin, Ireland
}

\begin{abstract}
Our propose is to build a network of virtual laboratories, based in a Virtual Closet that will contain all the elements and parts that are needed to build the various experiences available in a v-labs network (that we call Euronet-Lab).
\end{abstract}

To build this complex network we need to find a system that supports effectively this structure. This probably will be a enormous database of $v$-labs and independent elements, where will be possible sometimes to "recycle" some of the elements. This means "re-use" the same element several times in many experiences. To do this is necessary to have a structure that allows us to have several instances of the same element.

It's important that in our structure and virtual environment we can create several "images" of the same reality and this images can be used simultaneously in different circuits/experiments. This means that we can create several instances of the same element, to be used in different experiences and exercises.

Index Terms-Virtual Labs, CMS, Ontology, Remote Laboratory.

\section{INTRODUCTION}

The main problem that we want to solve is to find a structure that allow us to define a efficient and logical database that is completely able to support our virtual labs network.

This structure must permit to classify all the components and parts used in our experiences, and allow us to go get this elements/objects in a "Virtual Closet", that we must build and define, prior to anything else. We defend that this structure is an Ontology.

This "Virtual Closet" will be the base of our system to build the virtual experiments. And the structure that supports this "closet" ,must allow to use and define several instances of the same object that we go to use in different experiments that can exist and run at the same time.

One of the most important points to develop in this project is the "user interface" that will be used by all users of the system.

So in this point we will try to make and discuss a definition of user interface integration.

To integrate this user interface in all the EURONET LAB system, it is important to define logically and technically how we will make this integration.
So, the way we choose, is first to see what is now the "State of the Art" in this matter.

So, after consulting several documents in that area, we think that the most important point to see and study is the "Application Integration" with the developed ontology and the other components of the system that we will choose.

The application integration of the user interface is one of the most used techniques to connect the user with software applications.

There are several kinds of approach to solve this issue.

In this point we go discuss the definition of "user interface integration".

One of the most accepted models of integration interface is the model of Fowler [7],[10], and following that model we can define the three main layers:

1. The source layer

2. The business logic layer (or domain layer)

3. Presentation layer

Several authors derived from this classification. This leads to the simplest model of system integration;

- An integration layer can be placed in the top of each one of the layers, facilitating by this way the application integration in the 3 layers:

- Data layer (source layer)

- Business layer

- Presentation layer

To better understand the figure 1 we go present a little resume of the classification criteria for some authors:

- Amsden[8] introduces a variation of integration; one application may "involve" another, i.e. start it via access to the underlying operation system.

- Nisson [9] introduces a separation of integration on the user interface layer: that distinguishes the integration of "user interface parts" from the integration on the "screen handling" layer, for architectures as X Windows Scheifler and Gety [11],[10] defends that the implementation on User Interface components (UI components) is separated from the implementation of the display and the interaction with those component parts, which is what the author calls "screen handling". So, these authors Scheifler and Gaty [11],[10] propose two different strategies of integration on the UI components and also make changes on the screen handling layer. 
The classification of levels can be shown in the following diagram (Figure 1) of levels that show us the classification of levels made by several authors.

Linthicum [12] proposes and discusses several ways of "enterprise application integration", i.e. in the way of allowing the integration of different applications from several enterprises.

As main concept, Linthicum distinguishes two types of integration on the business logic level:

- Application interface integration

- Method integration

Let's see its differences with more detail:

- The application interface integration means that the application call methods from another one.

- Method integration implies the exchange of models and also more complex patterns of interaction between applications, going beyond simple method calls [10].

Other authors, Benatallah Nezad [13] provide an even finer-grained distinction of integration business layer. This comes besides the distinction of Linthicum's between application interface (called "function integration" by the authors). In this case they introduce the need for addictionally coordinating the message exchange itself (called basic coordination) as well as policies, such as privacy policies and quality of service agreements between systems. Also the authors introduce the communication layer as another layer of integration, thereby stressing that when integration distributed applications, the communication protocol heterogeneities must be overcome [14].

We must also take in consideration the main advantages and benefits of "Application Integration" on the "User Interface Layer".

There are two main benefits for performing application integration for the user interface level:

- Increasing the usability of software systems

- Reducing development efforts for those software systems.

We can see these benefits from two points of view:

- From the user, the end's user

- From the software engineer

From the end user point of view, we can say that any system that is integrated on a deeper level than the user interface, will come with an individually developed user interface [15] .

So the user will be confronted with a new unfamiliar user interface that requires time to the user to learn how to use this interface.

On the other hand, if we have a simple interface system the user easily learn how to work with the interface and is easier to use and doesn't require learning and adaptation time from the user.

From the software engineers point of view, reusing an existing interface, as opposed to developing a new inter face from the "zero point" means saving time and having less programming work.

It's important have in mind that the user interface is normally the most expensive part of a software system. The portion of development effort dedicated to the user

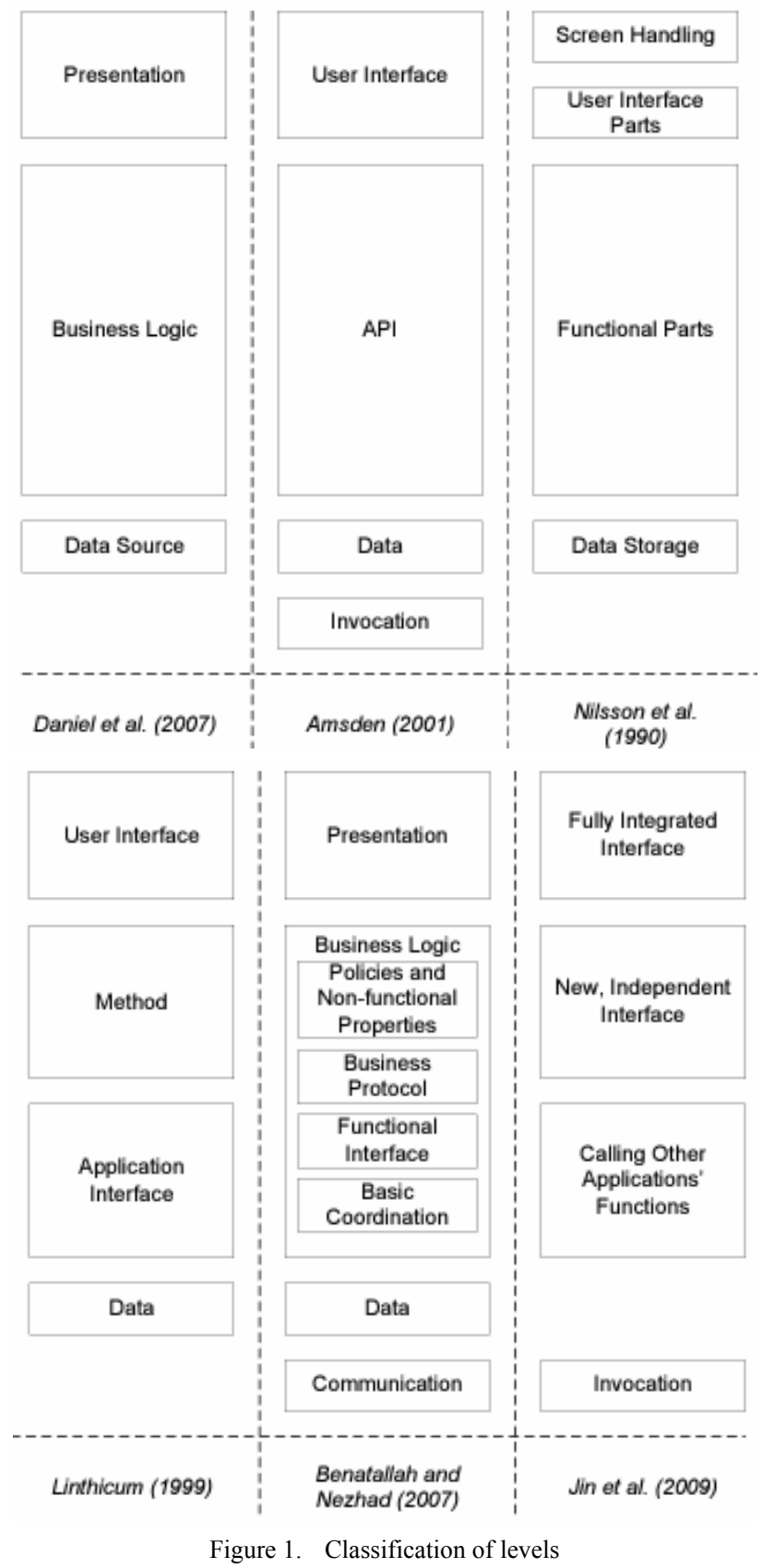

interface system in a project goes from $50 \%$ (Myers and Rosson, 1992) to 70\% [17] of the total development effort.

In resume we can say that without an approach for integration of the user interface level the degree of reuse will never be higher than $50 \%$. If we use UI integration, this action can therefore reduce development efforts of integrated software systems drastically.

Another very important aspect of the project to consider is; what are the requirements and challenges of Application Integration on the User Interface layer.

This application integration on the UI layers take us to some challenges. About these challenges [15] enumerates five requirements for UI integration approaches:

1. Definition of a common model and language for specifying components.

2. Definition of a model and language for specifying the integration. 
3. Create a support system for interaction and communication among the components.

4. Definition of a mechanism for visualizing the individual UI components.

5. Development of a mechanism for component discovery and binding.

A framework for the user interface integration has to have access to components to integrate in the ontology. Also is necessary to define a common model for those components, because is this model that will define how to access and control each component in the necessary actions to develop.

Typically, if we use a API, these components are API components. These API can be a high-level API, working at the level of business objects or, in another approach, can be a low-level API where the UI entities can be considered as buttons [15],[10].

Normally API's at both levels are very useful as way to facilitate meaningful user interface integration. For integrating the user interface components, the developer has to specify coordination of the different components and also, the kind of relations that exists among them. This can be done in any general purpose programming language $(\mathrm{C}++$ or Java) or in specialized languages.

To implement interactions between components, some mechanisms for communication between components has to be provided. This can be a message exchange facility, event-based communication, etc. Communication between components can be performed either directly or centrally mediated [15],[10]. As user interface programming itself is most often event-oriented but is normal too the use of event-based communication for UI integration as well [16]. In an integrated UI, the individual application user interface components have to be displayed on the screen. The framework can either split and delegate the display to individual components or performed a unified display, e.g. based on markup as HTML. The last issue is the discovery and binding of components. The most common solution applies when the set of applications to integrate is not fixed at the time when the development code of the system is built. In this case the components can be registered, for example in an online repository, and then sought, found and bound when necessary at run-time.

The work already done to build this system was the project and drawing of the system, and also the definition of the main branches and elements of the ontology to use.

We use Protégé Software as a tool to draw our ontology. So the definition of superclasses, sub-classes, and all the hierarchy is already build.

Also is already defined the concepts for each class build and also the properties of this concepts, also called is restrictions.

The contribution of this paper is explain and justify why, in our opinion, the better solution to implement this structure is an Ontology, and also define, and build all the objects and elements that we need in our system.

So we start to make an introduction, where we explain how to build an ontology, and what are the rules and steps that we must follow to correctly build the ontology we want to implement.

\section{Definition OF OnTOlogy:}

Generally we can define an Ontology as:

"A Ontology defines a common vocabulary for researches, and someone who need to share information in a domain". [1]

Ontologies are widely used in integration of application scenarios, most of the times in the data and business logic level.

In a frequently cited article "Ontologies: Principles, Methods and Applications", [18], point the usefulness of ontologies for promote inter-operability between IT systems.

The ontologies are proposed as a mean for "interlingua" for information exchange between applications.

The word "ontology" has its origin in the greek words:

ov $\tau$ $\xi$ ("being") and $\lambda \circ \gamma o \xi$ ("theory" or "science"). So, ontology is "being theory" or "being science", in fact it is a sub-area of philosophy that deals directly with the question of what existence actually is, and also it makes several categorization and organization of the existing things at a particular domain.

In computer science, "Ontology" is a formal model of a knowledge domain.

In philosophy area, ontology is used as singular word and refers a field of study, the computer science typically deals with many and various "ontologies" that are "formal models of a domain", but for the same domain can exist more than one model.

So there is a variety of definitions for ontologies in computer science:

Gruber [10],[19] says: "An ontology is an explicit specification of a conceptualization".

Guarino and Giaretta [10],[20] presents a more detailed definition as: "An ontology is a logical theory which gives an explicit, partial account of a conceptualization".

But Guarino [10],[21] defines an ontology as: "A set of logical axioms designed to account for the intended meaning of a vocabulary".

The authors outlined a new web semantic, which was not made up of texts that could only be understood by humans, but of information that could be processed by intelligent software's agents.

Figure 2 shows the so called "semantic web stack", that is a reference architecture which illustrates the language proposed by $\mathrm{W} 3 \mathrm{C}$ for implementing the semantic web.

The technological foundations on which the semantic web languages are built are the general-purpose extensible Markup Language, XML [10].

Figure 3 shows the 200 large datasets which are currently available as linked data.

Some rules can be used to express additional axioms that most ontology languages do not foresee. In the semantic web stack, various rule interchange formats to express rules defined with different individual rule languages, such as the Semantic Web Rule Language SWRL or the Rule Markup Language Rule ML [10]. Those rule languages allow for more flexible definitions than property chains in OWL2. 


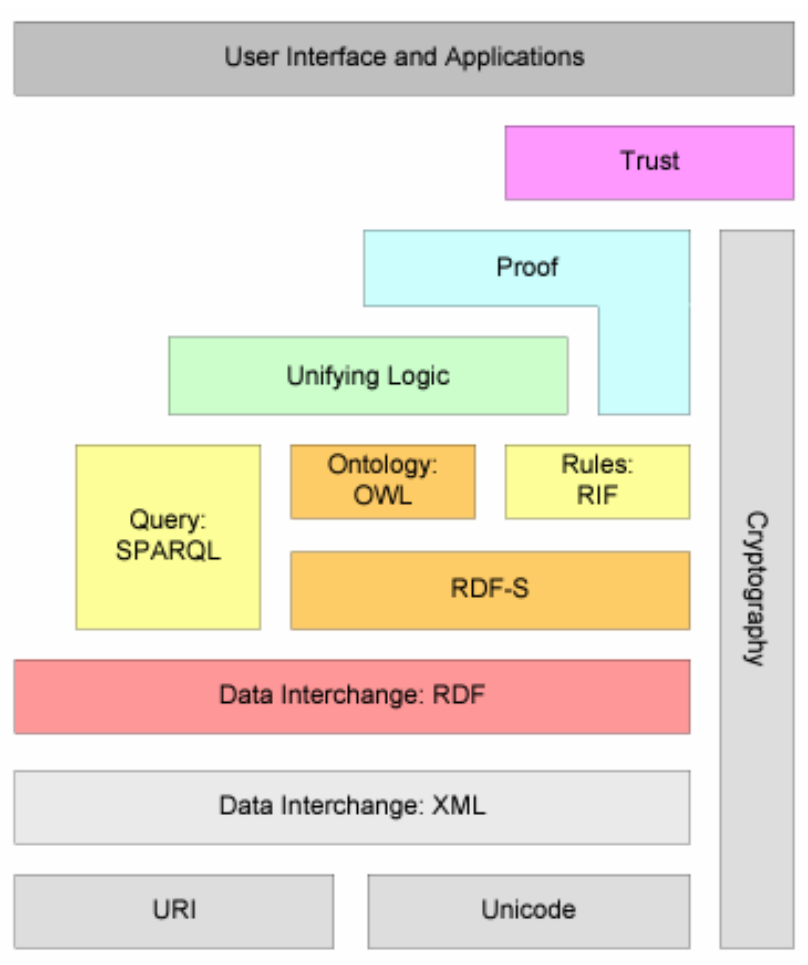

Figure 2. The semantic webstack

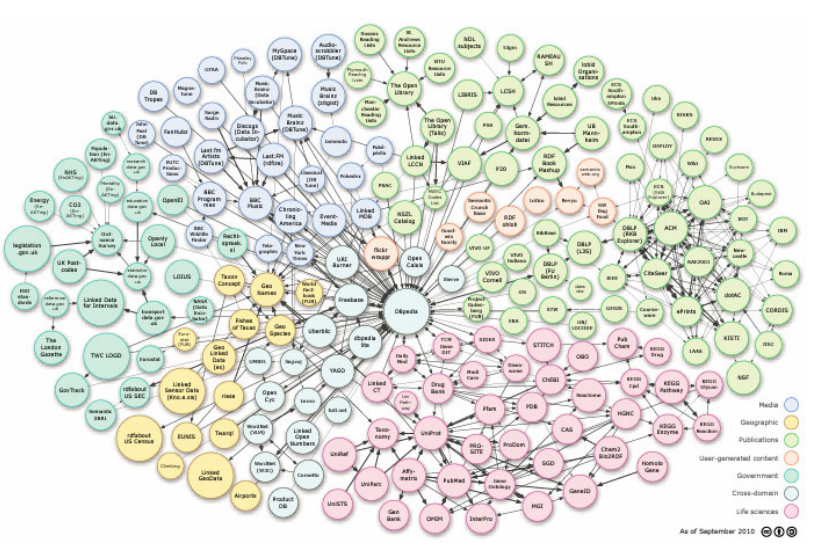

Figure 3. Aspect of the linked open datacloud

The Rule Interchange Format RIF provides an abstraction from those rule languages which is based in formal logic. It can be seen as an instantiation of the unified logic layer [10].

\section{QUERY SYSTEMS:}

To query the information contained in the semantic web or defined in RDF, ontologies and rules, various languages have been proposed, as we can see in the surveys made by Hease and others authors [10].

The query languages that were widely accepted for the ontologies are in RDF-based documents [10] are:

- SQL

- SPARQL

Apart from languages standardized or recommended by the World Wide Web Consortium, there are others that are used both in industry and universities.
The F-Logic is one of the most often used of these languages [10]. F-Logic which integrates ontology definitions and rules is one uniform language. Other than semantic web languages proposed by $\mathrm{W} 3 \mathrm{C}$, which follow the open world assumption, F-Logic uses closed world semantics. The basic building blocks of F-Logic ontologies are:

- Class and sub-class definitions.

- Relation definitions. Other than OWL, F-Logic does not support sub-relation definitions.

- Rules. Most of the definitions in F-Logic are rules. Like Prolog rules they consist of a head (i.e., what is stated to be true) and a body (the condition under which the head is true).

The next picture shows an example of an ontology in FLogic, which corresponds to the OWL example depicted in the code above.

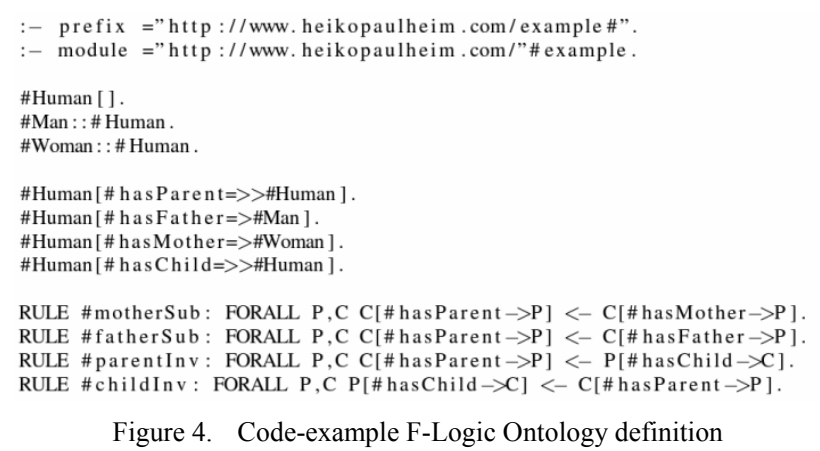

There are also other ontology languages as:

- KIF - Knowledge Interchange Format

- A Lisp - based notation for predicate logic

\section{TYPES OF ONTOLOGIES}

There may be various types of ontologies, developed and employed for different purposes. Various classification approaches have been presented, discussed and employed for different purposes. Various classification approaches have been discussed for comparing and distinguish these ontologies.

One of the first classifications of ontologies has been proposed by Heijst [10] and other authors who make their classification based in two properties:

- Their amount of structures or degrees of formality

- Their subject

Regarding their degree of formality, they distinguish:

- Termonological ontologies (that specify a list of terms and their meaning)

- Information ontologies (that specify the structure of data)

- Knowledge modeling ontologies (that makes a conceptualization of knowledge)

In 2001 lassila and McGuiness [10] provide a more detailed description and distinction between several types of ontologies shown and classified in the next picture: 


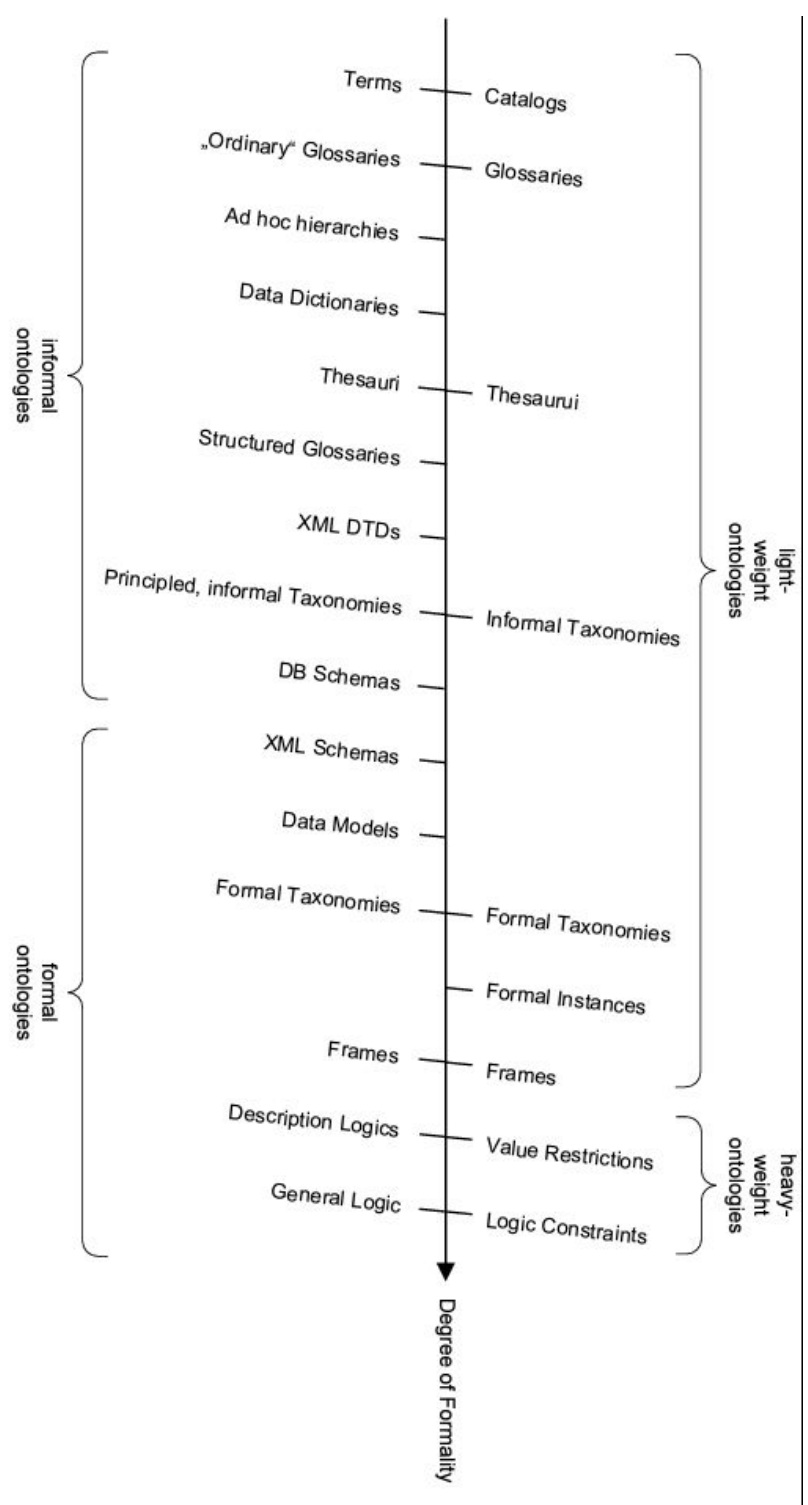

Figure 5. Ontology types based on the degree of formality

Glossaries - are catalogs that are enriched with descriptions for the terms.

Thesauri - contain additional relations between terms. Typically, those are relations such as "synonym of", "broader term than" or "narrower term than".

Informal taxonomies - arrange the terms in a hierarchy. An example is the concept hierarchies used by web shops.

Formal instances - are taxonomies that also esplicitly define instances.

Frames - are used to define relations between concepts, e.g. that each food products is made from ingredients.

Value restrictions - impose additional domain and range constraints on such frames, such as that only eatable substances can be used as ingredients for food products.

Logic constraints - are constraints that go beyond domain and range definitions, e.g. stating that categories of objects are disjoint.

It exist another wide level of classification of ontologies: referring the figure 5 , we can say that the first four (from the up) are sometimes referred as "informal ontolo- gies", and the last five (near the lower side) are "formal ontologies".

Another distinction that is several times used is "lightweight" and "heavyweight", where "heavyweight" includes value restriction and logic constraints and "lightweight" includes all the other categories, like show in the figure at left. As depicted in the figure, Uschold and Grünninger [10] further refine the classification given by Lassila and McGuiness [10] by adding the following classifications:

Ad hoc hierarchies - are even weaker than informal taxonomies. The hierarchies do not even intend to create correct is-a relations, but only group things that roughly belong together.

Data dictionaries - define complex types of data based on simple ones e.g., a date being composed by a day, a month, a year.

Structured glossaries - contain further relations between terms, e.g. synonym and antonym relations.

XTML DTD's - are meta-descriptions of XML documents. They define which elements in a XML file can exist and how they can be nested, showing the relations between all its elements. These nesting and relations provide informal, unnamed relations between nested elements.

Database schemas - describe tables in a database, their elements and their relations.

XML schemas - have the same purpose as XML DTD's but are more expressive.

Data models - refer to models that go beyond database schemas, e.g., UML-based models, possibly with additional constraints.

There are some other criteria to classify the ontologies; regarding its contents, Van Heijst [10] enumerates four types of ontologies:

- Domain Ontologies - define concepts of one specific domain.

- Generic Ontologies - define concepts that are general enough to be used across various domains.

- Application Ontologies - define concepts from a domain that are required for one application.

- Representation Ontologies - define the concepts that are used to define ontologies, i.e. they define concepts such as term or relation. They can also be considered as meta-ontologies (Ontologies used to define ontologies).

\section{REUSABILITY}

In "domain ontologies" and "generic ontologies" we registered a high level of reusability of the concepts and terms but not in "application ontologies", because normally they refer to a very particular domain of knowledge.

A similar distinction is used by Uschold and Jasper [10] The authors distinguish three meta-type (or meta-levels) of ontologies:

L0 - Operational Data - defines knowledge about instances such as "Lisbon is a city".

L1 - Ontologies - define the concepts and terms of a domain. Ontologies provide the vocabulary to define operational data. 
L2 - Ontology representation languages provide means for defining L1 ontologies.

The classification proposed by Guarino [10] distinguish ontologies by their level of abstraction and their usage as shown in figure 6.

Some of the ontology types resemble those in the classification by Heijst [10] and other authors as referred above.

Top-level ontologies of upper-ontologies - are equivalent to "generic ontologies". They contain general concepts that are useful across several domains, most often based on human perception of the world [10], proposed by Kiryakov and other authors.

Domain Ontologies - are equivalent to domain ontologies as are defined by Heijst and other authors[10].

Task Ontologies - define the activities of a task but without pointing a specific domain. For example scientific experiments contain hypotheses, measurements and evaluations, all of which can be defined agnostic to the actual domain of the experiment.

Application Ontologies - are equivalent to domain ontologies as defined by Heijst and other authors[10].

They identify the concepts defined in domain and task ontologies to define specific activities. This is done by stating which entities from the domain of that particular ontology plays which role in an activity defined in the task ontology.

The ontologies of the different levels are interconnected with specialization relationships. Thus, ontologies reuse definitions made by other ontologies on a higher level, therefore making them modular and comparable.

We choose to use an ontology, considering the main advantages is use, that are:

- Share common understanding of the structure of the information among people or software agents

- To enable reuse of domain knowledge

- To make domain assumptions explicit

- To separate domain knowledge from the operation knowledge

- To analyze domain knowledge

For the purposes for what we want use the ontology, we can consider that an Ontology is a formal explicit description of concepts in a domain of discourse.

The main elements of an Ontology are:

- Classes, sometimes called concepts

- Slots, are the properties of each concept describing various features and attributes of the concept. Sometimes slots are also called roles or properties

- Facets, are restrictions on slots, or even properties of slots, or restrictions of slots

An Ontology together with a set of individual instances of classes constitutes a knowledge base.

In reality, there is a fine line where the ontology ends, and the knowledge base begins.

In pratical terms, developing an Ontology includes:

- Defining the classes of the Ontology

- Arranging the classes in a taxonomic (sub-class - superclass) hierarchy

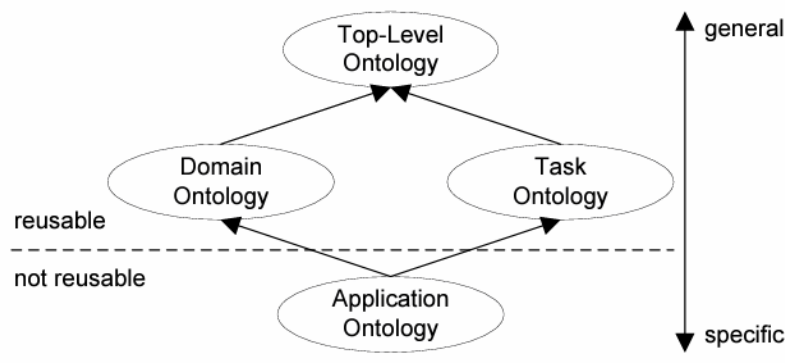

Figure 6. Classification of ontologies based on their level of abstraction

- Defining slots and describing allowed values for these slots

- Filing in the values for slots for instances

We can then create a knowledge base by defining individual instances of each classes filling in specific slot value information and additional slot restrictions.

To design correctly an ontology we must respect the following rules. These rules may seem rather dogmatic, but they can help to make correct design decisions in most of the cases where ontologies can be applied:

1. There is no one correct way to model a domain of knowledge - there are always several alternatives. The best way to implement our ontology depends on the application that we have in hands, and all the extensions of it that was possible to us to anticipate.

2. Continuous ontology developement process is necessary, and is an iterative process

3. Concepts in ontology should be very close to objects (physical or logic) and also close from the relationship that exist in the domain where we define the ontology.

4. Probably the most common is to define nouns (objects) or verbs (relationships) in sentences that describe your domain.

In a most detailed way, we can say that there are some steps that we must follow to define our ontology:

Step n. 1: Determine the domain and scope of our ontology.

To do this we must essentially to respond to the following questions:

- What is the domain that the ontology will cover?

- For what we going to use the ontology?

- For what types of questions the information on the ontology should provide answers?

- Who will use and maintain the ontology?

Step n. 2: Consider reusing existing ontologies:

It most always worth considering what someone else has done and checking if we can refine and extend existing sources for our particular domain and task.

Reusing existing ontologies may be a requirement if our system needs to interact with other applications that have already committed to particular ontologies or controlled vocabularies.

Step n. 3: Enumerate important terms in the Ontology:

It is useful it write down a list of all terms we would like either to make statements about or to explain to a user. 
Step n. 4: Define the classes and the class hierarchy:

These are several possible approaches in developing a class hierarchy (Uschold and Gruminger 1996):

- Top-down development process:

o Starts with the definitions of the most general concepts in the domain and subsequent specialization of concepts.

- Bottom-up development process:

o Starts with the definition of the most specific classes, the leaves of the hierarcly, with subsequent grouwing of these classes in more general concepts.

- Combination development process:

o This is really a combination of top-down and bottom-up approaches: we define the most salient concepts first and then generalize and specialize them appropriately.

None of these three methods is inherently better then any of the others. The approach to take depends strongly on the personal view of the domain and the situation in particular.

If a developer has a personal top-down view of the domain, then it may be easier to use the top-down approach.

However the combination approach is often the easiest way for many ontology developers, since the concepts "in the middle" tend to be the more descriptive concepts in the domain (Roch 1978).

Step n. 5: Define the properties of class-slots:

The classes done will not provide enough information to answer the competency questions.

Once we have defines some of the classes, we must describe the internal structure of concepts.

In general, there are several types of object properties that can become slots in an ontology:

If we take as example a Ontology about wines, we must considerer the following:

- "Intrinsic" properties (ex: flavor of a wine)

- "Extrinsic" properties (Name of the wine and area of production)

- "Parts" if the object is structures, these can be both physical and abstract "parts" (Indicated dishes to drink with)

- "Relationships to other individuals," these are relationships between individual members of the class and other items( maker of the wine, type of greap"

Note: All the subclasses of a class inherit the slot of a class.

Step n. 6: Define the facets of the slots:

Slots can have different facets describing the value type, allowed values, the number of values (cardinality), and other features of the value the slot can take.

Some normal common facets are:

Slot Cardinality: Defines how many values a slot can have some details:

- Some systems distinguish between single cardinality (Allowing at least one value) and multiple cardinality (allowing any number os values)
- Some systems allow the specification of a maximum and a minimum cardinality to describe the number of slots more that a slot must have at least $\mathrm{N}$ Values.

Slot value Type, they have some possible types that corresponds to the common variable data types:

- String

- Number (Float or integer)

- Boolean (yes-no flag`s)

- Enumerated (list of specific allowed values)

o Instance (instance-type slots allows the definition of relationship between individual.

- Instance must also define a list of allowed classes from which the instances can come.

The classes to which a slot is attached or the classes which property a slot describes, are called the domain of the slot.

We can define the range of a slot as the allowed classes for slots of type instance.

Some systems allow restricting the range of a slot when the slot is attached to a particular class.

Step n. 7: Create instances:

The last step to create an ontology is creating individual instances of classes in the hierarchy. To accomplish this step we must do the following "sub-steps":

1. Choosing a class

2. Creating as individual instance of that class

3. Filling the slot values

One of the objectives of this work is to build and define libraries of reusable knowledge components, (like RLO reusable learning objects in SCORM specification) and also Knowledge - based services than can be invoked over networks; to achieve this objective the most indicated structure to define and describe all the elements of a virtual laboratory as parts and components is an ontology, by the above reasons exposed.

Also we can say that an ontology permit to describe in a very detailed way the components / elements of the "virtual closet", with all is details and features.

So formally we can say that an Ontology is the statement of a logical theory.

In a pragmatic way we can say that an Ontology defines the vocabulary with which queries and assertions are exchanged between systems that communicate in the v-labs network.

Ontological commitments constitutes agreements that should be used as shared vocabulary in a coherent and consistent way.

As conclusion of this introduction we can say: "an ontology is a particular system of categories accounting for a certain vision of the world. This system does not depend of a particular language. A shared ontology need only describe a vocabulary for talking about a domain, where as a knowledge base may include the knowledge needed to some a problem or answer arbitrary about a domain".

\section{OUR Solution to the Problem / OUR Ontology:}

To implement and build the ontology we go use the software "Protégé" that is a tool specially developed to build ontologies. 
Protegé is a software that allows easily to build ontologies respecting all the rules that we define in our system.

In Protegé we define what will be the classes and also we can define all the relations between them.

In our particular case the "root" or Master-class of our ontology is "LABORATORY" (figure 7).

From there we define three main classes:

- Experiment

- Real Component

- Virtual Component

Essentially the ontologies used in this field of knowledge using semantic web technologies. We can define, accordinng to the $\mathrm{W} 3 \mathrm{C}$, "The Semantic Web provides a common framework that allows data to be shared and reused across application, enterprise, and community boundaries." Tim Berners-Lee defines the Semantic Web as "a web of data that can be processed directly and indirectly by machines."

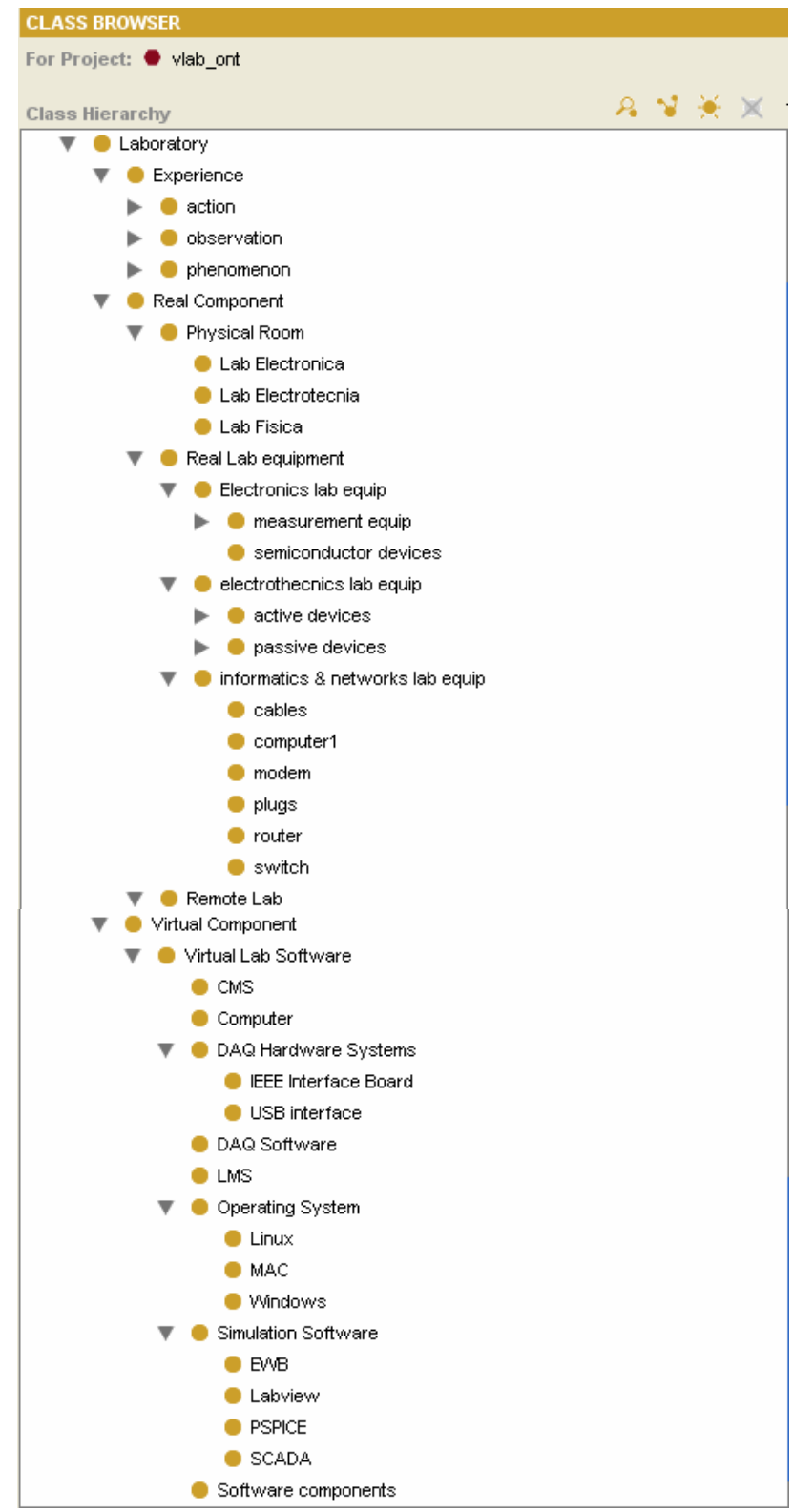

Figure 7. EuronetLab Ontology definition
All the laboratories are composed by experiments, that we can define as:

"An experiment is the smallest enclosed unit of an online laboratory. It provides also the execution of virtual or real experiments to observe the behavior and output of a system. An online laboratory consists of one or more experiments in different fields of science and engineering". Ref. [1]. Also normally, associated to an online laboratory, there must be other learning resources like a laboratory tutorial and lecture notes to provide the theoretical background necessary to carry out an experiment. Therefore, it is necessary to provide a variety of additional documents and references. Ref. [1]. So is very important to have as support of all this system a CMS or a e-learning platform that interconnect the build ontology with the vlabs proposed in the "virtual closet".

The CMS , LMS or a e-learning platform allows the existence and organization of all this pedagogical and technical pedagogical contents and supports.

The proposed network as the following block-diagram:

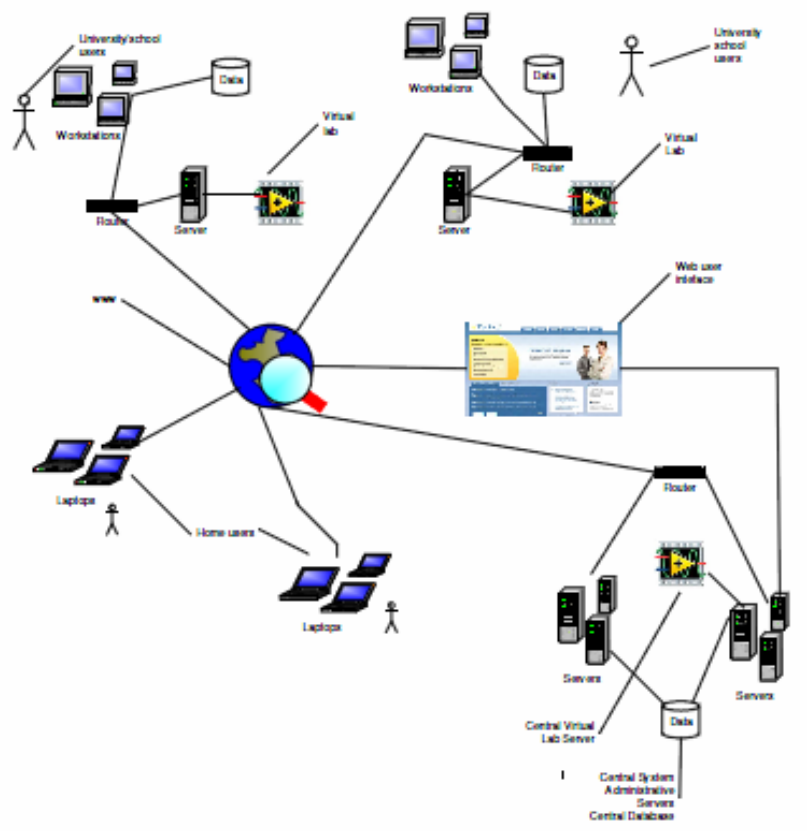

Figure 8. EuronetLab main structure

In this network we have three main actors:

- Teachers

- Students and Researchers

- Administrative staff

A login, forms and database must be created using ASP or PHP that allows students to:

- Schedule an experiment in a certain lab;

- Verify available labs and in which universities or institutes are located;

- Verify the experiments they have done, their grade and comments from their teachers;

- Read or review pedagogical contents that support the different experiments.

The teachers should be able to:

- Send pedagogical contents;

- Review contents and materials; 
- Evaluate the students' Works;

- Communicate with the students using email, chat, video-conference in order to give orientations and clarify subjects.

The administrative staff should support all the nontechnical issues and administrative issues derived from the communication between universities, institutions, teachers, researchers and students.

This is the way that we think that this V-labs network, the "Euronet - Lab" should work. Ref. [5]. Ref [6].

\section{FUTURE DEVELOPMENTS}

The following action to take in this project is to build a prototype system that interconnects the build ontology with the v-labs network and the LMS databases that support the administrative parts of the system as shown in figure 9 .

\section{REFERENCES}

[1] Abul K.M. Azad et al., Internet Accessible Remote Laboratories: Scalable E-Learning Tools for Engineering and Science Disciplines, Engineering Science Reference (an imprint of IGI Global) 701 E. Chocolate Avenue Hershey PA 17033 Tel: 717-533-8845 Fax: 717-533-8661 E-mail: cust@igi-global.com Web site: http://www.igi-global.com.

[2] Gruber, T.R., 1993a. Toward Principles for the Design of Ontologies Used for Knowledge Sharing N. Guarino \& R. Poli, eds. International Journal of Human-Computer Studies, 43(5-6), pp.907928. Available at: http://inkinghub.elsevier.com/retrieve/doi/10.1006/ijhc.1995.1081

[3] Gruber, T.R., 1993b. Toward Principles for the Design of Ontologies Used for Knowledge Sharing N. Guarino \& R. Poli, eds. International Journal of Human-Computer Studies, 43(5-6), pp.907928.

$$
\text { Available }
$$
http://linkinghub.elsevier.com/retrieve/doi/10.1006/ijhc.1995.1081

[4] Noy, N.F. \& Mcguinness, D.L., 2000. Ontology Development 101: A Guide to Creating Your First Ontology. Development, 32(1), pp.1-25. Available at: http://citeseerx.ist.psu.edu/viewdoc/download?doi=10.1.1.136.508 5\&amp;rep=rep1\&amp;type=pdf.

[5] Cordeiro, R., Fonseca, J. M., \& Donellan, A. (n.d.). A Cloud Based Laboratory Environment.

[6] Cordeiro R., Passos, H., ed. Virtual Labs in the E-Learning Context as Tools of Collaboration Work. EDULEARN 09. 2009: Barcelona.

[7] FlecharM (2010) HypeCycle for Application Development.http://www.gartner.com/DisplayDocument?id=1412014,acce ssed April12th,2011.

[8] AmsdenJ(2001) Levels Of Integration-Fiveways you can integrate with the EclipsePlatform. http://www.eclipse.org/articles/ArticleLevels-Of-Integration/levels-of-integration.html,accessed April 12th,2011.

[9] Nilsson EG, NordhagenEK,OftedalG(1990) Aspects of systems integration.In:ISCI'90:Proceedingsof the first international conference on systems integrationon Systems integration'90, IEEEPress,pp 434-443

[10] Paulheim, Heiki, Ontology-based Application Integration, ebooks: www.e-books.com, accessed August 17 th , on 2012.

[11] Scheifler RW,GettysJ (1986) The X WindowSystem.ACM Transactions on Graphics5(2):79-109 http://dx.doi.org/10.1145/ 22949.24053

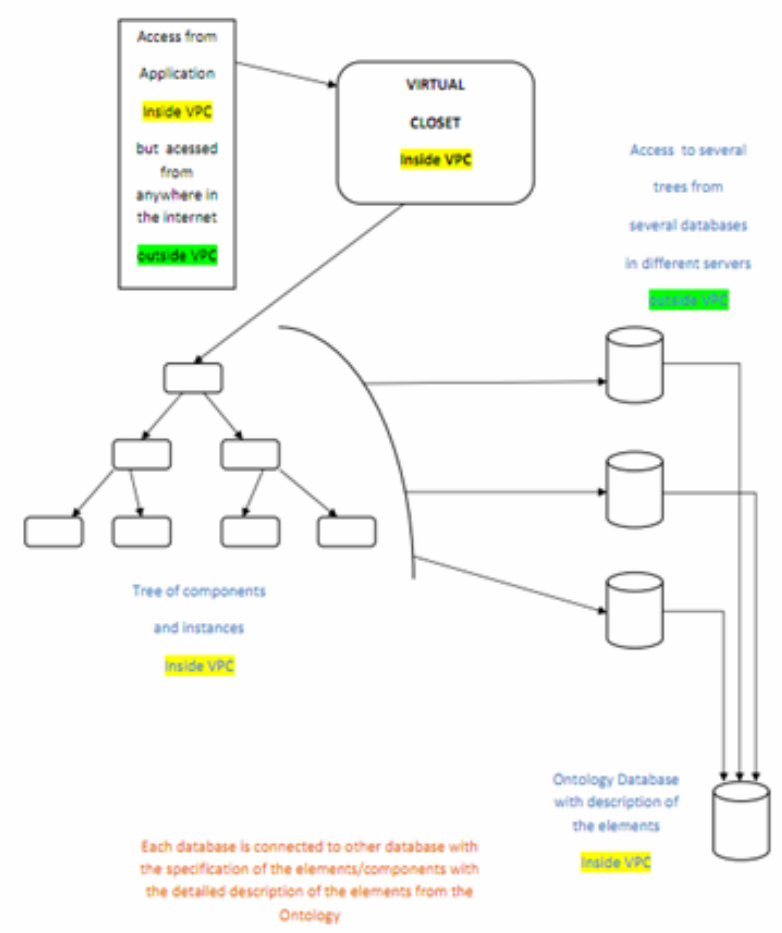

Figure 9. EuronetLab database and VPN structure

[12] LinthicumDS(1999) Enterprise Application Integration. Addison Wesley

[13] BenatallahB,NezhadHRM(2007) Service Oriented Architecture: Overviewand Directions. In: B" orgerE,CisterninoA(eds) Advances in Software Engineering, Springer,LNCS,vol5316,pp 116-130

[14] Rebstock M,Fengel J,Paulheim H(2008) Ontologies-based Business Integration. Springer

[15] Daniel F,Matera M(2008) Mashing Up Context-Aware Web Applications: AComponent-Based Development Approach.In: WISE'08: Proceedings of the 9th international conferenceon Web Information Systems Engineering,Springer,LNCS, vol5175,pp250263

[16] WestermannU,Jain R(2007) Toward a Common Event Model for Multimedia Applications.IEEE MultiMedia14(1): 19-29 http://dx.doi.org/10.1109/MMUL.2007.23

[17] SergevichK A,ViktorovnaG V (2003)From an Ontology-Oriented Approach Conception to User Interface Development. International Journal"Information Theories and Applications"10(1):89-98

[18] Uschold M,Gruninger M (1996) Ontologies:Principles, Methods and Applications.Knowledge Engineering Review11:93-136 http://dx.doi.org/10.1017/S0269888900007797

[19] Gruber T R (1995) Toward Principles for the Design of Ontologies Used for Knowledge Sharing. International Journal HumanComputer Studies 43(5-6):907-928 http://dx.doi.org/10.1006/ ijhc.1995.1081

[20] Guarino N,Giaretta P (1995) Ontologies and KnowledgeBases: Towards a Terminological Clarification. In:MarsNJI(ed) Towards Very Large Knowledge Bases:Knowledge Building and Knowledge Sharing,IOSPress,Amsterdam, pp25-32

[21] Guarino N,Welty CA(2009)An Overview of Onto Clean. In:(StaabandStuder,2009),chap10,pp 201-220 


\section{AUTHORS}

C.C. Raúl, Raúl Cordeiro Correia is with the Instituto Politécnico de Setúbal - Escola Superior de Tecnologia de Setubal, Largo Defensores da Republica $n^{\circ}$ 1, 2910-470 Setúbal, PORTUGAL. E-mail: raul.correia@estsetubal.ips.pt

M. F. José, José Manuel Fonseca is Professor in the Electrical Engineering Department of the Universidade Nova de Lisboa, Campus da FCT/UNL, 2829-516 Caparica, Portugal. Email: jmrf@,fct.unl.pt
D. Andrew, Andrew Donnellan is a lecturer in the Department of Electronic Engineering, Institute of Technology, Tallaght, Dublin 24, Ireland. Email: andrew.donnellan@ittdublin.ie

This work was supported in part by IPS - Instituto Politécnico de Setúbal - escola Superior de Tecnologia de Setúbal. It is an extended and modified version of a paper presented at the International Conference on Remote Engineering \& Virtual Instrumentation (REV2012), held at University of Deusto, Bilbao, Spain, July 4-6, 2012. Received 9 September 2012. Published as resubmitted by the authors 14 November 2012. 